\title{
Gene Expression Profiles for Predicting Metastasis in Breast Cancer: A Cross-Study Comparison of Classification Methods
}

\author{
Mark Burton, ${ }^{1,2}$ Mads Thomassen,, ${ }^{1,2}$ Qihua Tan, ${ }^{1,2}$ and Torben A. Kruse ${ }^{1,2}$ \\ ${ }^{1}$ Research Unit of Human Genetics, Institute of Clinical Research, University of Southern Denmark, Sdr. Boulevard 29, \\ 5000 Odense C, Denmark \\ ${ }^{2}$ Department of Clinical Genetics, Odense University Hospital, Sdr. Boulevard 29, 5000 Odense C, Denmark \\ ${ }^{3}$ Institute of Public Health, University of Southern Denmark, J. B. Winsløws Vej 9B, 5000 Odense C, Denmark \\ Correspondence should be addressed to Mark Burton, mark.burton@ouh.regionsyddanmark.dk
}

Received 25 August 2012; Accepted 2 October 2012

Academic Editors: M. A. Kon and K. Najarian

Copyright ( $) 2012$ Mark Burton et al. This is an open access article distributed under the Creative Commons Attribution License, which permits unrestricted use, distribution, and reproduction in any medium, provided the original work is properly cited.

Machine learning has increasingly been used with microarray gene expression data and for the development of classifiers using a variety of methods. However, method comparisons in cross-study datasets are very scarce. This study compares the performance of seven classification methods and the effect of voting for predicting metastasis outcome in breast cancer patients, in three situations: within the same dataset or across datasets on similar or dissimilar microarray platforms. Combining classification results from seven classifiers into one voting decision performed significantly better during internal validation as well as external validation in similar microarray platforms than the underlying classification methods. When validating between different microarray platforms, random forest, another voting-based method, proved to be the best performing method. We conclude that voting based classifiers provided an advantage with respect to classifying metastasis outcome in breast cancer patients.

\section{Introduction}

The analysis of high-dimensional gene expression datasets has posed new computational challenges. These datasets have, for example, in breast cancer research, been applied to develop classifiers predicting metastasis outcome, disease recurrence, or breast cancer survival. Some of the classification methods most frequently applied to microarray data are logistic regression $[1,2]$, support vector machines (SVM) [312], neural networks (NNET) $[1,13]$, random forest (RF) $[1$, 12], and classifiers based on voting [1]. However, few studies have systematically compared the predictive performance of such methods using microarray gene expression datasets on breast cancer. In their studies, method comparisons have been done within the same datasets by, for example, 10-fold cross-validation, leave-one-out cross-validation, or hold-out procedures [14-18], addressing prediction of relapse within a 5 -year period [14, 16, 19], or molecular subtype classification [15]. Furthermore, even fewer studies have compared crossstudy validation between classification methods within the field of breast cancer research. Two studies addressed ERpositivity and molecular subtype classification [20,21], while another tested prediction of relapse within a 5 -year period in a small group of 19 independent patients [22].

This study compares the performance of seven classification methods belonging to four different categories for predicting metastatic outcome in lymph negative breast cancer patients, which have not been treated with adjuvant systemic therapy. The classification methods used included an ensemble decision tree model (random forest), regression (logistic regression), four support vector machines and a neural network. To address various degrees of variation for such tasks, the comparisons were done either within the same dataset (internal) or between different datasets (external). Within the same dataset model building and classification were performed using 10 -fold cross-validation. Across datasets the comparisons were done in two ways. The first is in which the validations are conducted between studies using the same microarray platform (classifiers developed from an Affymetrix dataset and validated on an independent Affymetrix dataset), while the second encompasses validations across studies with different platforms (classifiers developed from an Agilent dataset and validated on an independent Affymetrix dataset). Furthermore, we 
TABLE 1: Overview of datasets used.

\begin{tabular}{|c|c|c|c|c|c|c|c|c|c|c|}
\hline Dataset & Chip & $\begin{array}{l}\text { Probes } \\
(K)\end{array}$ & Patients & Outcome & Treatment & $\begin{array}{l}\text { Define } \\
\text { genes }\end{array}$ & $\begin{array}{c}\text { Internal } \\
\mathrm{CV}\end{array}$ & $\begin{array}{c}\text { External } \\
\text { validation } \\
\text { train } \\
\end{array}$ & $\begin{array}{c}\text { External } \\
\text { validation } \\
\text { test }\end{array}$ & Reference \\
\hline Amsterdam & Agilent/Rosetta & 25 & $295, \mathrm{~N}^{+}, \mathrm{N}^{-}$ & $\mathrm{DM}$ & $\begin{array}{l}\text { None, et, } \\
\text { ct }\end{array}$ & $\sqrt{ }$ & & & & {$[31]$} \\
\hline $\begin{array}{l}\text { Amsterdam } \\
\text { (AM) } \\
\text { (subset of the } \\
\text { above) }\end{array}$ & Agilent/Rosetta & 25 & $151, \mathrm{~N}^{-}$ & $\mathrm{DM}$ & None & $\sqrt{ }$ & $\sqrt{ }$ & $\sqrt{ }$ & & {$[31]$} \\
\hline Rotterdam (RO) & $\begin{array}{c}\text { Affymetrix } \\
\text { HG-133A }\end{array}$ & 22 & $286, \mathrm{~N}^{-}$ & $\mathrm{DM}$ & None & $\sqrt{ }$ & $\sqrt{ }$ & $\sqrt{ }$ & & {$[28]$} \\
\hline HUMAC & $\begin{array}{c}\text { Spotted } \\
\text { oligonucleotides }\end{array}$ & 29 & $60, \mathrm{~N}^{-}$ & $\mathrm{ME}$ & None & $\sqrt{ }$ & & & & {$[4]$} \\
\hline Huang & $\begin{array}{l}\text { Affymetrix } \\
\text { 95av2 }\end{array}$ & 12 & $52, \mathrm{~N}^{+}$ & $\mathrm{RE}$ & $\mathrm{ct}$ & $\sqrt{ }$ & & & & {$[27]$} \\
\hline Sotiriou 2003 & Spotted cDNA & 7.6 & $99, \mathrm{~N}^{+} / \mathrm{N}^{-}$ & $\mathrm{RE}$ & et, ct & $\sqrt{ }$ & & & & {$[24]$} \\
\hline Sotiriou 2006 & $\begin{array}{c}\text { Affymetrix } \\
\text { HG-133A }\end{array}$ & 22 & $179, \mathrm{~N}^{+} / \mathrm{N}^{-}$ & $\mathrm{DM}$ & et & $\sqrt{ }$ & & & & {$[25]$} \\
\hline Uppsala & $\begin{array}{c}\text { Affymetrix } \\
\text { HG-133A+B }\end{array}$ & 44 & $236, \mathrm{~N}^{+} / \mathrm{N}^{-}$ & $\mathrm{DF}$ & $\begin{array}{l}\text { None, ct, } \\
\text { et }\end{array}$ & $\sqrt{ }$ & & & & {$[6]$} \\
\hline Stockholm & $\begin{array}{c}\text { Affymetrix } \\
\text { HG-133A+B }\end{array}$ & 44 & $159, \mathrm{~N}^{+} / \mathrm{N}^{-}$ & $\mathrm{RE}$ & $\begin{array}{c}\text { None, ct, } \\
\text { et }\end{array}$ & $\sqrt{ }$ & & & & {$[23]$} \\
\hline $\begin{array}{l}\text { TRANSBIG } \\
\text { (TR) }\end{array}$ & $\begin{array}{c}\text { Affymetrix } \\
\text { HG-133A }\end{array}$ & 22 & $147, \mathrm{~N}^{-}$ & $\mathrm{DM}$ & None & & & & $\sqrt{ }$ & {$[32]$} \\
\hline Mainz (MA) & $\begin{array}{c}\text { Affymetrix } \\
\text { HG-133A }\end{array}$ & 22 & $200, \mathrm{~N}^{-}$ & $\mathrm{DM}$ & None & & & & $\sqrt{ }$ & {$[33]$} \\
\hline
\end{tabular}

examined the effect of combining the classification results on each sample by the seven methods into one final classification determined by majority voting, and performances compared by internal and external validation as well.

\section{Materials and Methods}

2.1. Datasets Used in This Study. The following eight datasets were used for either defining the gene features and or training purposes in the further study: samples from the studies [23-27] and samples from the Gene Expression Omnibus(GEO-) series GSE2034 [28], GSE4796 [4], and GSE3494 [6] (Table 1). A subset of 151 node-negative samples from the dataset by van de Vijver (AM) and the entire GSE2034 dataset [28] (abbreviated RO) were used for classifier development in the further study (Table 1). The following datasets were used as independent testing sets: the node-negative samples from GSE7390 [29] (abbreviated TR) and the GSE11121 dataset [30] (abbreviated MA) (Table 1).
2.2. Dataset Processing. The eight datasets above were downloaded and directly used for identification of rank-significant genes. Following this identification, the four datasets: AM, $\mathrm{RO}, \mathrm{TR}$, and MA were all standardized to have mean zero and standard deviation one. Calculations and classification were all conducted using the $\mathrm{R}$ free package. For random forest, logistic regression, support vector machines, and neural network we used the randomForest, glm, e1071, and nnet packages, respectively.

2.3. Identification of Cross-Study Rank-Significant Features. To determine which genes should be used to build gene expression classifiers, we used the eight publicly available datasets mentioned above, which were used in our two previous studies $[34,35]$. This was done by applying the microarray meta-analysis described in [34], upon the individual gene expression values of each individual probe/gene in the eight datasets. This method ranks each individual gene in each dataset according to its signal-to-noise ratio, calculates the 
gene's mean rank across datasets, and determines if this mean rank is significantly high or low, according to a significance cutoff at FDR $\leq 0.05$.

2.4. Classifier Building. The features within each training dataset were ranked according to their random forest variable importance measure. For each feature, this value reports the standardized drop in prediction accuracy when the classlabels are permuted [36]. For each feature, this rank was used for model building by subsequently adding one feature at a time in a "top-down" manner. To avoid creating bias, during gene selection and training of the final classifier, and on classification performance, we used ten-times repeated 10fold cross-validation accuracies as a performance measure, as this metric has previously been shown to give an excellent bias-variance balance [37]. In this study, the models were developed to achieve the best mean sensitivity and specificity thus forcing the overall accuracy to give a balanced sensitivity and specificity. Seven different classification methods were used for model building which included: random forest (RF) [36], logistic regression, SVM with a radial- (R-SVM), a linear (L-SVM), polynomial (P-SVM), or a sigmoid-based kernel (S-SVM) [38], and a neural network with a single hidden layer (NNET). The voting approach is described in detail below. As all classification methods have hyperparameters, we optimized these parameters during model building using a grid-like search of parameter combinations. In random forest, we optimized the number of trees in the forest ( $n$ tree) from settings of 2000, 3000, 4000, and 5000 trees, and the number of subselected predictors for node splitting (mtry) with settings of: $1,0.5 \cdot \sqrt{(\text { number of features })}, 1$. $\sqrt{(\text { number of features })}, 2 \cdot \sqrt{(\text { number of features })}$, and total number of features. In all support vector machines, the slack variable penalizing cost parameter $(\mathrm{C})$ was optimized using settings of $0.01,0.1,1$, and 10 , and the $\gamma$-parameter, controlling the spreading of samples in feature space, with the settings of $0.001,0.01,0.1$, and 1 , and for P-SVM also the polynomial degree using degrees of 2,3 , and 4 .

2.5. Voting. The voting procedure can be regarded as a metamodel, where a sample is first fed to be predicted by each of the respective classification methods. These predictions are next fed to the final metamodel, combining each of these predictions into a final classification determined by majority voting.

The voting procedure, at the level of internal dataset prediction, consisted of two steps. In the first step, each sample is classified ten times during $10 \times 10 \mathrm{CV}$, meaning that each sample is given 10 votes for classification within each classification method. To prevent ties, the nine first votes were used for class decision. In the second step the final votes from each of the seven classification methods are combined into one vote, thus creating the cross-classification voting result.

During external validation, every sample is classified once by each of the seven classification methods. The voting classification for each sample is determined by the winning class assigned by the seven classification methods (voters).
2.6. Classification Performance Assessment. We compared the bAcc, defined as the mean of sensitivity and specificity, of classifiers at two levels either internal or external. Internal performance was determined by the 10 -times repeated 10 fold cross-validation classification accuracies. External performances obtained through transferring the trained classifier from the training sets to classify each of the independent samples are reported. In external validation, two different situations were examined: (1) between similar (RO on TR or MA) and (2) different microarray platforms (AM on TR or MA), covering Affymetrix-based classifiers validated on an Affymetrix dataset and Agilent-based classifiers validated on another Affymetrix dataset, respectively.

2.7. Endpoint/Outcome Definition. The outcome is defined as metastasis after time of diagnosis. As this study addresses outcome classification, we did not consider the time-toevent component or censoring, due to the fact that survival analysis sometimes can be misleading when considering classification, and because transformation of time-to-event into a binary outcome can blur prediction of the classes [39].

2.8. Comparison of External Validation Performance. There is to our knowledge no standard statistics for comparing classifier performance on unbalanced datasets using the balanced accuracy as a performance measure. Therefore, in order to test the significance of the performance difference between the classification methods (defined as a significant difference between correct predictions using method A versus using method B), we used a repeated downsampled binomial test approach consisting of five steps. (1) The classifiers classification results upon the entire test data were initially converted into a balanced test result by downsampling. Downsampling obtains a class-balanced dataset from an imbalanced dataset by removing a subset of randomly selected samples from the majority class, where the number of samples removed equals the difference in sample size between the major and the minor class. In this study the majority class is the nonmetastasis class; (2) the number of samples correctly classified by one classification method but incorrectly by the other classification method and vice versa is counted; (3) the significance of the difference in these counts is determined using a binomial $\chi^{2}$-test; $(4)$ the $P$-value of this test is stored. The steps 2 to 4 are repeated 1000 times; (5) from the 1000 tests, the median $P$ value is reported as the statistical significance impact between the two compared methods.

\section{Results}

3.1. Features and Classifiers/Models. In this study the classifiers were developed to predict metastasis outcome using full follow-up time. To make the classifiers globally applicable and robust, we identified genes being significantly associated with outcomes across eight different studies using different microarray platforms and originating from different populations. These eight datasets are referred to as the "feature definers" (FD) (Table 1). In the further analysis, two of the 
FD datasets were used as training sets. The first, Rotterdam (RO), is an Affymetrix-based dataset containing 286 samples, and the second, Amsterdam (AM), is a node-negative subset of 151 samples from the entire FD-Amsterdam dataset (Table 1). Two independent datasets, not used for feature selection or classifier development, were used as test sets. These comprise the TRANSBIG (TR) and Mainz (MA) datasets, which are based on the Affymetrix platform and consist of 147 and 200 samples, respectively.

As a preliminary feature selection step, we identified genes being significantly associated with outcomes across eight different studies using a rank-based method (as described in Section 2). This method led to identification of 519 rank-significant genes. By matching the 519 ranksignificant genes and those present in AM, RO, TR, and MA, these genes were reduced to 283 (Figure 1) and were thus used for classifier building. The list of 283 genes is shown in Supplementary Table 1 in Supplementary Material available online at doi:10.1100/2012/380495.

In order to build the models, the 283 features within each training dataset were ranked according to their random forest variable importance measure (Figure 2). For a given feature, this measure reports the standardized drop in prediction accuracy when the class labels are permuted [36]. This rank was then used for model building by subsequently adding one feature at a time in a "top-down" forward manner (Figure 2).

\subsection{Comparison of Classification Methods: Internal Validation} Performance. To reduce variability, and complexity and to keep validation parameters as constant as possible, the performance of the classifiers was tested within the same dataset by a ten-times repeated 10-fold cross-validation (Figure 2). This validation scheme partitions the training data into 10 nearly equal-sized folds. Subsequently, 10 iterations of training and validation are performed. During each of these iterations a different fold of the training data is left out for validation and the remaining are used for learning. The mean accuracy of all 10-folds validated is thus the 10-fold crossvalidated $(10 \times 10 \mathrm{CV})$ accuracy of the model. By repeating this process 10 times a more robust and unbiased estimation of the performance is obtained. In our study, the balanced accuracy (bAcc), defined as the mean of sensitivity and specificity, was used as a performance measure. It should be noted that the individual classification performances are artificially elevated due to information leakages, caused by $\mathrm{AM}$ and RO being used for primary feature selection, and that the entire AM and RO datasets are used for importance ranking prior to cross-validation. However, the differences between the individual classification method performances are assumed, unaffected by these leakages.

The performances within the AM and RO by each classification method were combined, and the mean performance calculated. The classifiers based on NNET had the best performance achieving a mean $10 \times 10 \mathrm{CV}$ bAcc of 78\%, followed by S-SVM, L-SVM, R-SVM, P-SVM, RF, and LR achieving mean $10 \times 10 \mathrm{CV}$ bAcc of $74.1 \%, 72.1 \%$, $71.6 \%, 70.9 \%, 69.4 \%$, and $68.0 \%$, respectively (Figure 3 and Supplementary Table 2). The significance of these
The 8 feature defining datasets

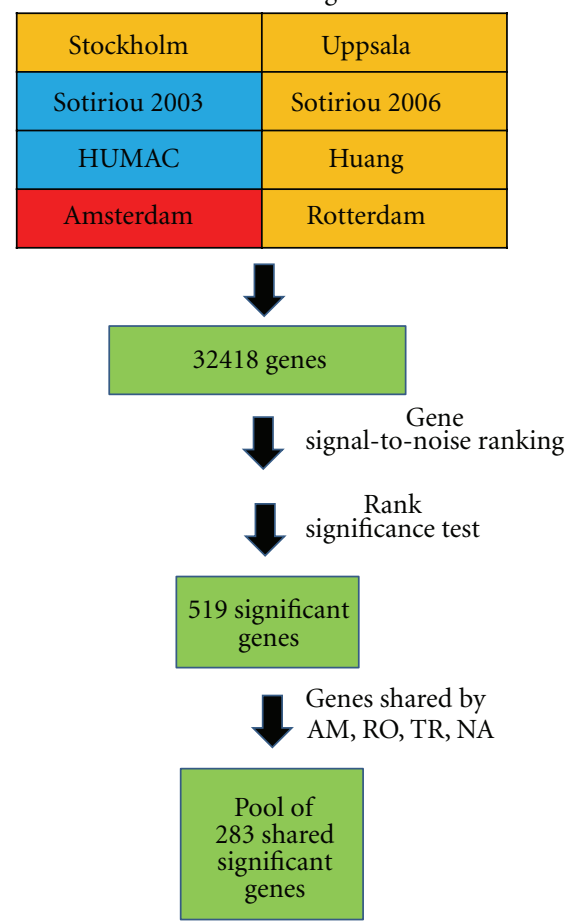

FIgURE 1: Feature selection. Eight breast cancer gene expression datasets (feature defining datasets), covering 32418 genes, were used to define a list of rank significant genes. Datasets using the Affymetrix platform, spotted oligonucleotides, and the Agilent platform are colored orange, blue, and red, respectively. These genes were first ranked within each of the eight datasets according to their signal-to-noise ratio, and their across dataset mean rank calculated. This mean rank was significance tested as described in Section 2, resulting in a list of 519 rank significant genes. These 519 genes were reduced to a pool of 283 genes shared by the two training sets (AM and $\mathrm{RO}$ ) and the testing sets (TR and MA), used in the further study.

differences was tested using the down-sampling statistical test described in Section 2, showing that NNET significantly outperformed $\operatorname{RF}(P=0.011)$, LR $\left(P=1.2 e^{-5}\right)$, LSVM $(P=0.027)$, and P-SVM $\left(P=6.3 e^{-4}\right)$. NNET only borderline significantly outperformed R-SVM $(P=0.07)$ and S-SVM $(P=0.10)$. Furthermore, S-SVM also performed significantly better than RF $(P=0.049)$, LR $\left(P=9.0 e^{-5}\right)$, and P-SVM $(P=0.049)$, and R-SVM outperformed RF $(P=0.018)$. No significant performance difference was found when comparing the other classification methods.

We next combined the cross-validated results by the seven methods into a voting procedure. This led to a mean $10 \times 10$ CV bAcc of $86.9 \%$, which significantly outperformed all the seven underlying classification methods: RF $(P=$ $\left.1.1 e^{-19}\right)$, LR $\left(P=1.7 e^{-18}\right)$, R-SVM $\left(P=4.2 e^{-12}\right)$, L-SVM $\left(P=1.4 e^{-14}\right)$, P-SVM $\left(P=8.9 e^{-16}\right), \operatorname{S}-\operatorname{SVM}\left(P=5.8 e^{-11}\right)$, and NNET $\left(P=2.6 e^{-4}\right)$ (Figure 3 and Supplementary Table 2).

3.3. External Validation Performance between Similar Microarray Platforms. The performance of the classifiers was validated in independent datasets based on the same 
Internal validation
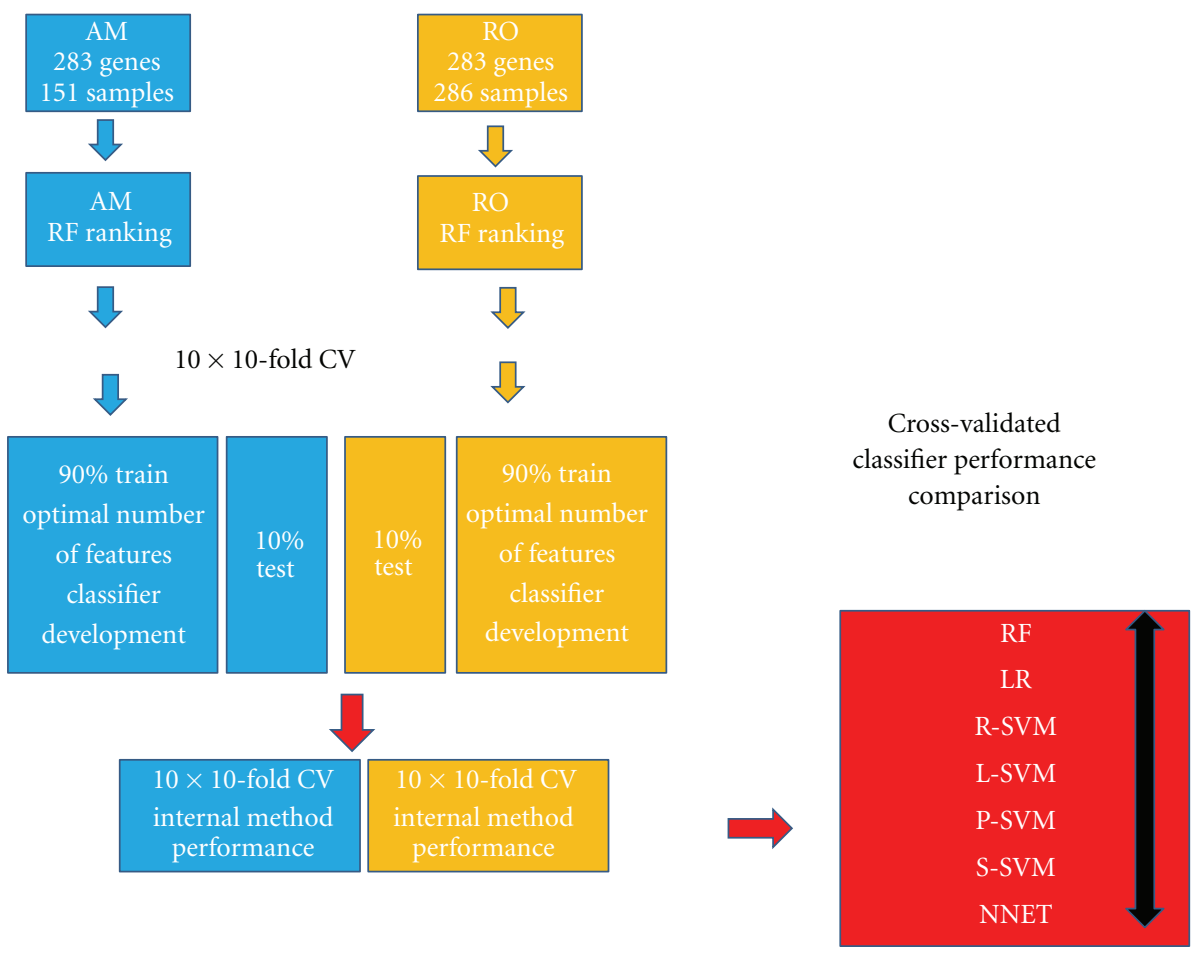

FIGURE 2: Internal validation procedure. The two datasets, AM (blue) and RO (orange) composed of the 283 rank-significant genes and 151 or 286 samples, respectively, were used for internal performance evaluation. These datasets were first individually used to rank each feature by their random forest variable importance value (RF ranking). These ranks were separately used for selecting the optimal number of features by adding one feature using the same classification method, using a 10-times repeated 10-fold cross-validation procedure. The $\mathrm{AM}$ and RO 10-times cross-validation results using the same classification method were combined, and the mean classification performance of each method was compared.

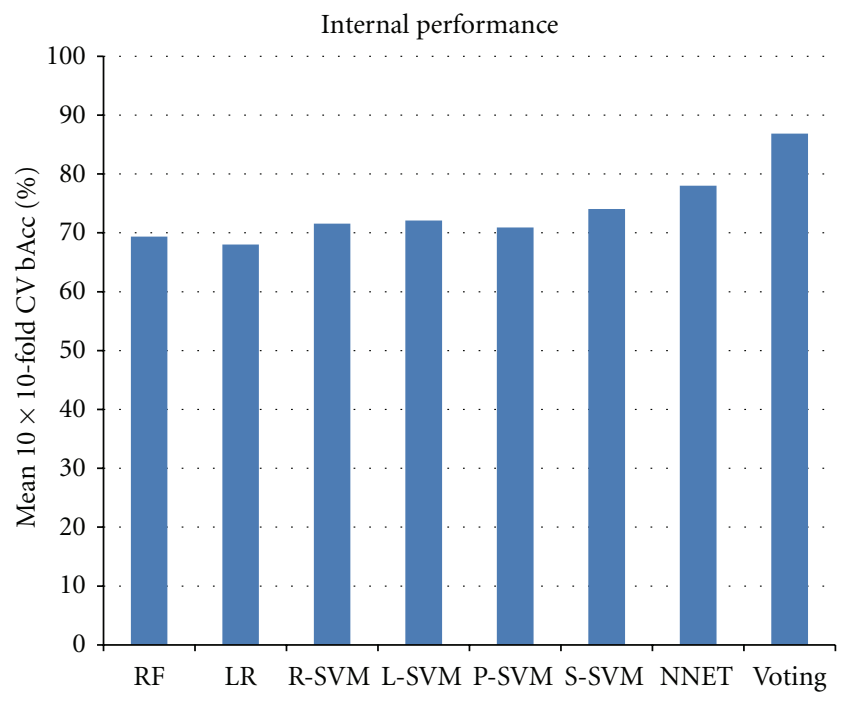

FIGURE 3: Internal validation performance. Shown in blue histograms are the mean 10 -times repeated 10 -fold cross-validation balanced accuracy performance (bAcc) within the two training datasets: AM and RO. Methods used are random forest (RF), logistic regression (LR), support vector machines with a radial (R-SVM), linear (L-SVM), polynomial (P-SVM), sigmoid kernel (S-SVM), a neural network with a single hidden layer (NNET), or cross-method voting (Voting).
TABLE 2: Number of features in the models.

\begin{tabular}{lcc}
\hline Dataset & AM & RO \\
\hline Method & $(n=151)$ & $(n=286)$ \\
\hline RF & 21 & 21 \\
LR & 5 & 11 \\
R-SVM & 20 & 25 \\
L-SVM & 8 & 11 \\
P-SVM & 4 & 7 \\
S-SVM & 17 & 35 \\
NNET & 21 & 16 \\
\hline
\end{tabular}

$\mathrm{AM}$ and $\mathrm{RO}$ are the Amsterdam and Rotterdam training sets and $n$ shows the number of samples in the respective datasets. Methods used are as follows: RF: random forest, LR: logistic regression, R-, L-, P-, and S-SVM: support vector machine with a radial basis function, linear, polynomial, or sigmoid kernel, and NNET: neural network with a single hidden layer.

microarray platform (Affymetrix), which covers the validation of RO-based classifiers on the TR and MA test data (Figure 4), which contained between 7 to 35 features (Table 2). In this setting, the entire classifiers developed in the training set, using the features and rules associated with the classifiers, were used to classify the independent samples in the entire test sets, and the performance is defined as the mean test accuracy in TR and MA. 

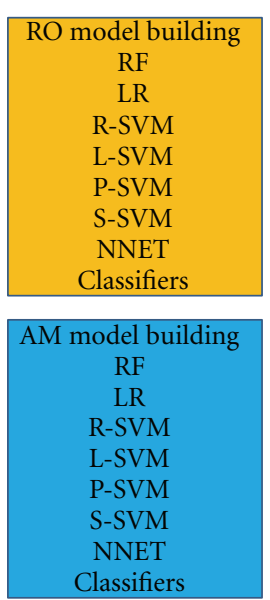

Classifier external validation procedure

\section{Between similar platform validatio}
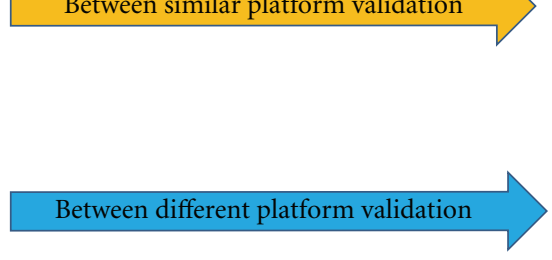

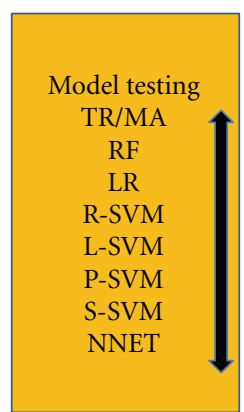

FIGURE 4: The procedure for external validation of classifiers. External classifier validation. Two datasets were used for training (AM and RO), and two others for testing (TR and MA). Datasets based on the Affymetrix and Agilent platforms are shown in orange and blue, respectively. RO and AM classifiers were used for evaluating external validation of classifiers developed from datasets using similar platform and using different microarray platforms, respectively. The models built in RO were tested in TR and MA and their mean performance calculated. This was done for all classification methods and compared. The same was done for testing AM models.

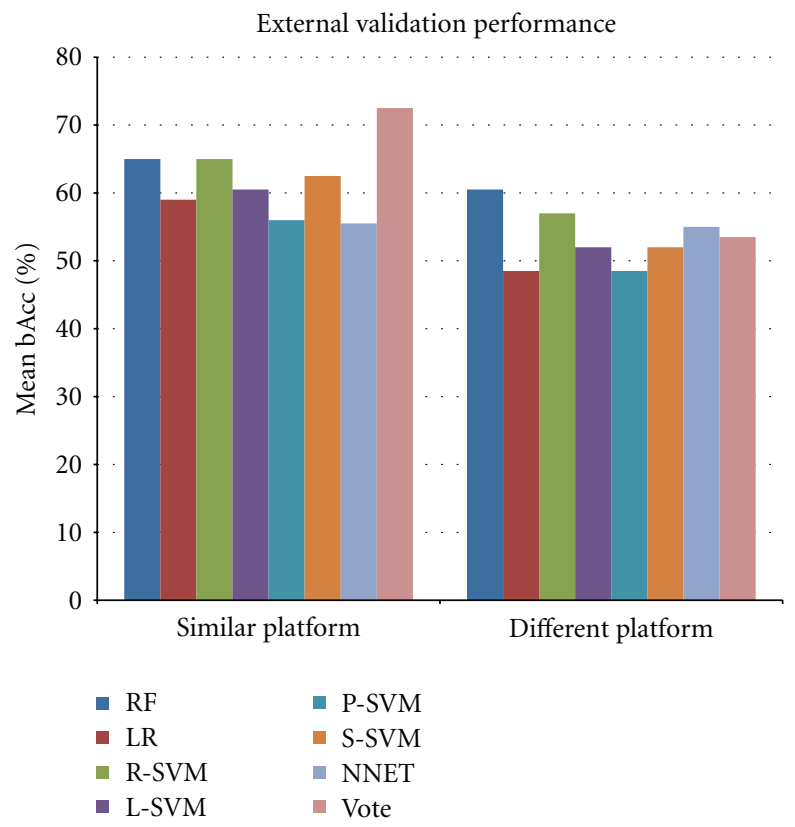

FIGURE 5: External validation performance. External classifier performance. The histogram shows the mean testing performance when classifiers are validated in test sets based on similar platforms or different platforms as from which they were developed. Each bar represents the mean balanced accuracy by random forest (RF), logistic regression (LR), support vector machines with a radial (R-SVM), linear (LSVM), polynomial (P-SVM), sigmoid kernel (S-SVM), a neural network with a single hidden layer (NNET), or cross-method voting (VOTE), respectively.

RF and R-SVM had the best external classification performance achieving a mean bAcc of $65 \%$, while NNET had the poorest performance (55.5\% mean bAcc) (Figure 5). RF performed significantly better than LR $(P=0.0085)$, LSVM $(P=0.026), \operatorname{P}-S V M(P=0.0057)$, and NNET $(P=$ $0.0012)$, and R-SVM also performed significantly better than
LR $(P=0.0064)$, L-SVM $(P=0.025)$, P-SVM $(P=0.013)$ and NNET $(P=0.0025)$ (Figure 5).

The voting procedure increased the performance to a $72.5 \%$ mean bAcc and significantly outperformed the seven underlying methods: $\operatorname{RF}\left(P=6.1 e^{-5}\right)$, $\operatorname{LR}\left(P=1.5 e^{-8}\right)$, R-SVM $(P=0.00026)$, L-SVM $\left(P=6.0 e^{-8}\right)$, P-SVM 
$\left(P=2.1 e^{-7}\right)$, S-SVM $\left(P=1.9 e^{-6}\right)$, and NNET $\left(P=4.7 e^{-7}\right)$ (Figure 5). Detailed overview of the individual validation results is shown in Supplementary Table 3.

3.4. External Validation Performance between Different Microarray Platforms. The performance of the classifiers was finally validated in independent datasets (Affymetrix) based on a different microarray platform from the one used by training data (Agilent) and covers the validation of AMbased classifiers on the TR and MA test data. These classifiers contained 4 to 21 features (Table 2). As in the case of the between-similar-platform validation, the entire classifier developed in the training set, using the features and rules associated with the classifiers, was used to classify the independent samples in the entire test sets (Figure 4), and the performance is defined as the mean test accuracy in TR and MA.

Comparison of classifiers developed on an Agilent dataset and validated on an Affymetrix dataset revealed that the mean classification performances based on RF had the best performance amongst the seven methods, achieving a mean bAcc of $60.5 \%$, while the poorest performances were achieved by the LR- and P-SVM classifiers, which obtained only $48.5 \%$ bAcc (Figure 5). RF performed significantly better than the other six methods: LR $\left(P=3 e^{-4}\right)$, R-SVM $(P=0.038), \mathrm{L}-\mathrm{SVM}(P=0.0012), \mathrm{P}-\mathrm{SVM}\left(P=9.8 e^{-5}\right)$, S-SVM $(P=0.0015)$, and NNET $(P=0.032)$ (Figure 5$)$. In contrast to the between-similar-platforms validations, the voting procedure only obtained a mean bAcc performance of $53.5 \%$, which was a borderline significantly inferior to RF $(P=0.059)$ (Figure 5). Detailed description of the individual between different platforms validation results is shown in Supplementary Table 4.

\section{Conclusion and Discussion}

This study compared seven classification methods and a voting procedure ability to predict metastasis outcome in lymph node-negative breast cancer patients. The results showed that during internal assessment and external validationmethods based on voting had the best performance.

Our study first compared the internal performance within a single dataset and showed that NNET had the best performance followed by the support vector machines, while RF and LR had the worst performances. This implies that at least for prediction of metastasis outcome within the same dataset-NNET and support vector machines displays superiority. This finding agrees well with other studies using cross- or hold-out procedures for performance comparisons. For example, one study comparing the performance of eight different classification methods showed that NNET and SVMs in general perform better than the other six methods for predicting outcome in eight different cancer microarray datasets [15]. Several studies confirm our finding of RF inferiority when using cross-validation $[17,18,40]$. Interestingly, a study conducting algorithm comparison on microarray gene expression based drug signatures showed that NNET and R-SVM had the best performance when tested in the most heterogeneous datasets [41]. As the datasets used in our study are likely to be very heterogeneous, due to the nature and etiology of breast cancer, the superior performances of NNET and support vector machines could reflect the ability of these particular methods to distinguish outcome in such complex datasets.

Combining the classification results by each method into classification based on voting significantly increased the internal performance. The finding of voting superiority in the internal validations suggests that voting would be valuable when applied to datasets having a combination of limited technical variation (due to using same protocols and platforms) and biological heterogeneity. Although the patients in our study are limited to being node negative, they may still be very heterogeneous due to the existence of various breast cancer molecular subgroups and the disease etiology. Voting may therefore reduce the variation associated with this biological heterogeneity. This is in line with the above-mentioned study, showing that some classification methods are more suitable for prediction tasks in complex datasets [41].

Our finding of voting superiority agrees with four other studies: one using multiple different feature extraction methods in combination with SVM for gene microarray classification showed that using a voting-based method across all the examined combinations achieved a better 10 -fold crossvalidated classification performance compared to any single combination [33]; a second study showed that an SVM-based ensemble outperformed single SVM for microarray data classification [42]; a third study comparing the performance of principal component discriminant classifiers either with or without voting using cross-validation applied on a simulated dataset a leukemia microarray gene expression dataset, a Gaucher serum proteomics dataset and a grape extract metabolomics dataset, also showed that voting had a better performance than the nonvoting method [43]; a fourth study comparing the performance of single models to combined models in thirteen diverse microarray datasets, which included predictions of estrogen receptor positivity and complete pathological response to chemotherapy in breast cancer, found that the majority of combined multiple models had a better classification performance than the single models [21]. Furthermore, our findings also agree with a study by Taylor and Kim who, by splitting their original datasets into training and test parts, showed that voting based on nearest mean voters was a top performing method with respect to classification on lung or prostate cancer data. In contrast to our results, RF was found to perform equally well as the mean voter [44]. However, this discrepancy is likely caused by the difference in classification tasks. In contrast to our results, Statnikov and coworkers found that SVM-based ensemble/voting methods perform similar or worse compared to SVM nonensemble/voting methods, when tested on ten different human gene expression datasets by 10 -fold cross-validation. However, these comparisons were primarily based on multicategory classification [45].

In the second experiment, we investigated the classifiers performance when tested in an external dataset based on a similar microarray platform. In this setup, RF and 
R-SVM achieved the best performances, both significantly outperforming four of the five remaining classification methods. Furthermore, the voting procedure significantly outperformed the seven underlying classification methods. This suggests that every classifier in the voting committee agrees on most of the samples that are predicted correctly and that the majority of voters do not make the same misclassifications. The finding that voting and RF have the best performances could be explained by these methods' ability to reduce the cross-study prediction variance, without simultaneously increasing prediction bias [37]. Only a limited number of studies have compared the cross-study performance of multiple classification methods. A study by Tan and Gilbert compared the performance of single C4.5 classifiers with the voting-like $\mathrm{C} 4.5$ bagging and boosting classifiers on gene expression data cancer classification. In four of the experiments, an independent dataset measured on similar platforms was used for testing. Interestingly, one of the results found that bagging and boosting performed better than single $\mathrm{C} 4.5$ classifiers when predicting relapse within a 5-year period in a small group of 19 independent breast cancer patients, achieving $88.7 \%, 88.7 \%$, and $75 \%$ bAcc, respectively [46]. These voting results are higher than the mean voting performance achieved in our two test sets (72.5\% bAcc). This could be due to three factors: (1) the training and testing sets used by Tan and Gilbert originate from the same population (The Netherlands), and the sample preparations and gene expression measurements were performed using the same protocols; (2) the classification task also differs. It might be easier to predict relapse within a 5 -year period than predicting if a patient would ever metastasize; (3) the voting methods used also differ. Another study deployed a committee of neural networks for gene expression based leukemia subclassification using three gene expression datasets measured on the same microarray platform. The study used a first dataset for feature selection, a second for network training and committee development, and a third independent test set for validating the committee. When compared to the performance by each of the underlying classifiers in the committee, the committee neural networks proved to perform better or equally well in the final testing set [47].

In the third experiment, the trained classifiers were externally validated on datasets based on a different microarray platform. With this setup, the performances dropped dramatically. This suggests that the data distributions in the Agilent and Affymetrix datasets are dissimilar. This is likely caused by biological and technical variation. The fact that the training and test samples originate from two different patient populations could make the data distributions dissimilar. The technical variation may originate from several sources, for example, the size of the oligonucleotides used, probe coverage, labeling, cross-hybridization, and detection limits by the scanner. Furthermore, the two platforms use different strategies for measuring the same RNA quantity. On the Agilent platform, this quantity is measured as the ratio of fluorescence intensities between a sample and a reference at each spot on the array, while the Affymetrix platform uses single channel measurements for a collection of probe sets covering one gene, which are therefore not comparable. To circumvent this obstacle, we standardized the datasets. However, this standardization seemed not to be sufficient for avoiding a drop in performance by all the classification methods used. Therefore, it is likely that the data distributions of the training and test sets are very heterogeneous thus hampering the external application of the classifiers.

Although all classification methods experience a drop in performance when validating between datasets measured on different platforms, the results showed that RF remained the strongest method and significantly outperformed the six other methods. Surprisingly, the voting procedure performed poorly when validated on data measured on a different microarray platform and was a borderline significantly outperformed by RF. This is probably due to randomness by each method/voter. The finding of RF performing better than the cross-method voting procedure suggests that when tested on datasets using a different microarray technology for gene expression measurement, voting procedures based on the same classification algorithm, in the case of random forest being a collection of decision trees, are more advantageous than voting procedures based on diverse classification methods. This implies that RF compared to voting is more capable of reducing the prediction variance associated with validation across studies and platforms. Therefore, in a situation when validating between different microarray platforms and where voting is outperformed, an approach called bagging might prove advantageous. Bagging uses voters consisting of multiple classifiers developed by bootstrap resamplings from the same dataset and based on the same classification method (decisions trees in the case of random forest) [48]. Thus, bagged SVM, LR, and NNET might be considered ideal for cross-study-cross-platform validations. RF may also be powerful, as the method is based on multiple decision rules, which might be better at segregating a complex data structure. This situation is in line with a study showing that molecular classification of cancer achieves better or similar performance as other classification algorithms, when using decision rules based on a single gene or a gene pair [49].

In the literature, there has been a limited number of studies comparing the performance of multiple classification methods, applied to across the dataset and microarray platform validations. One study by Yoshida compared a nearest template prediction method (NTP) with CART (single decision tree method), weighted voting, SVM, and knearest neighbor classification (k-NN) across datasets using Agilent datasets for training and Affymetrix datasets for testing [20]. For prediction of estrogen receptor positivity in breast cancer, NTP had the best performance, while SVM had the worst performance. For predictions of breast cancer molecular subtypes, SVM had the best performance in two of three testing sets used for this purpose, while NTP had the best performance in the third dataset. The worst performing methods were achieved by CART and k-NN [20]. In our study, SVM was not a top performing method for crossplatform testing. These differences are likely due to two factors: first, the study by Hoshida did not apply the entire classifier to the test sets, but only the list of genes defined 
by the training datasets. This list was used to train and test a classifier in the validation dataset; second, the study addresses completely different classification tasks compared to our study.

Our results showed that when validation is applied between two datasets of similar or different microarray platforms, LR and NNET were among the poorest performing methods.

The general poor performance of LR could be due to several factors. First, a strong LR model is frequently composed of predictors being highly univariate significant and remains significant in the multivariate model. The fact that the list of 519 rank-significant genes defined by the eight feature definer datasets was reduced to a pool of 283 genes could have led to the exclusion of some highly significant genes, due to the only reason that they were not shared by the AM, RO, TR, and MA datasets, thus impairing the possibility for development of a stronger model. This could explain the poor performance by LR classifiers developed by the individual AM and RO datasets; second, an LR model requires a large sample size for providing robust maximum likelihood parameter estimation. Although the training datasets contain 151 and 286 samples, these sample sizes may not be sufficient for developing a strong model if some of the highly discriminative genes are absent; third, LR models rely on the assumption that there is no colinearity between the variables, meaning that the variables/features should be independent from each other. This assumption may be violated if predictors in a logistic model consist of, for example, gene expression features, some of which could be coregulated, thus leading to colinearity and weakening the model; finally, LR is sensitive to outliers. As we have not removed any samples from our datasets, and the possibility of outlier presence thus could be evident, this could also hamper the predictive power of the LR models.

The finding of NNET had a high internal 10-fold cross validation performance but a weak external validation performance could suggest that the NNET classifiers are not very generable. Another explanation could be that the transfer function used by the neural network was a sigmoid function, which is identical to that used in logistic regression, thus leading to some of the weaknesses observed in logistic regression, although the parameter estimation in neural networks is not conducted by maximum-likelihood but by a gradient descent algorithm. Interestingly, a study has compared the classification performance of four different single hidden layer feedforward neural networks on three microarray gene expression cancer dataset, showing that an SVD-neural classifier based on a tansig activation function and using single value decomposition for parameter estimation had a better performance compared to the three other methods and that this classifier outperformed support vector machines, principle component analysis classifiers, and Fisher discriminant analysis classifiers [50]. This implies that using another neural network type could achieve a better performance when applied for external validation in datasets based on similar or different microarray platforms.

In conclusion, voting-based classifiers provided an advantage with respect to classifying metastasis outcome in breast cancer patients. When testing was performed within the same dataset or between datasets using similar microarray platforms, combining class decisions by multiple classification methods significantly increased the classification performance. Random forest, a voting-like method, proved to be the strongest method when testing was performed in datasets based on a different microarray platform.

\section{Conflict of Interests}

The authors declare that they have no competing interests.

\section{Acknowledgments}

This work was funded by the Danish Ministry of Interior, the Danish Strategically Research Council and DBCG-TIBCAT, the Clinical Institute at the University of Southern Denmark and the Human Microarray Center associated with the Department of Clinical Genetics at the Odense University Hospital. Furthermore, the authors acknowledge authors and people making the microarray datasets used in this study publically available.

\section{References}

[1] E. Karlsson, U. Delle, A. Danielsson et al., "Gene expression variation to predict 10 -year survival in lymph-node-negative breast cancer," BMC Cancer, vol. 8, article 254, 2008.

[2] H. Y. Chuang, E. Lee, Y. T. Liu, D. Lee, and T. Ideker, "Network-based classification of breast cancer metastasis," Molecular Systems Biology, vol. 3, article 140, 2007.

[3] Q. Tan, M. Thomassen, and T. A. Kruse, "Feature selection for predicting tumor metastases in microarray experiments using paired design," Cancer Informatics, vol. 3, pp. 133-138, 2007.

[4] M. Thomassen, Q. Tan, F. Eiriksdottir, M. Bak, S. Cold, and T. A. Kruse, "Prediction of metastasis from low-malignant breast cancer by gene expression profiling," International Journal of Cancer, vol. 120, no. 5, pp. 1070-1075, 2007.

[5] R. Sabatier, P. Finetti, N. Cervera et al., "A gene expression signature identifies two prognostic subgroups of basal breast cancer," Breast Cancer Research and Treatment, vol. 126, no. 2, pp. 407-420, 2011.

[6] L. D. Miller, J. Smeds, J. George et al., "An expression signature for $\mathrm{p} 53$ status in human breast cancer predicts mutation status, transcriptional effects, and patient survival," Proceedings of the National Academy of Sciences of the United States of America, vol. 102, no. 38, pp. 13550-13555, 2005.

[7] M. H. van Vliet, R. Fabien, H. M. Horlings, M. J. van de Vijver, M. J. T. Reinders, and L. F. A. Wessels, "Pooling breast cancer datasets has a synergetic effect on classification performance and improves signature stability," BMC Genomics, vol. 9, article 375, 2008.

[8] M. Thomassen, Q. Tan, F. Eiriksdottir, M. Bak, S. Cold, and T. A. Kruse, "Comparison of gene sets for expression profiling: prediction of metastasis from low-malignant breast cancer," Clinical Cancer Research, vol. 13, no. 18, part 1, pp. 5355-5360, 2007.

[9] N. Servant, M. A. Bollet, H. Halfwerk et al., "Search for a gene expression signature of breast cancer local recurrence in young women," Clinical Cancer Research, vol. 18, no. 6, pp. 1704$1715,2012$. 
[10] T. Zeng and J. Liu, "Mixture classification model based on clinical markers for breast cancer prognosis," Artificial Intelligence in Medicine, vol. 48, no. 2-3, pp. 129-137, 2010.

[11] M. Garcia, R. Millat-carus, F. Bertucci, P. Finetti, D. Birnbaum, and G. Bidaut, "Interactome-transcriptome integration for predicting distant metastasis in breast cancer," Bioinformatics, vol. 28, no. 5, pp. 672-678, 2012.

[12] R. Díaz-Uriarte and A. S. de Andrés, "Gene selection and classification of microarray data using random forest," BMC Bioinformatics, vol. 7, article 3, 2006.

[13] L. J. Lancashire, D. G. Powe, J. S. Reis-Filho et al., "A validated gene expression profile for detecting clinical outcome in breast cancer using artificial neural networks," Breast Cancer Research and Treatment, vol. 120, no. 1, pp. 83-93, 2010.

[14] A. Statnikov and C. F. Aliferis, "Are random forests better than support vector machines for microarray-based cancer classification?" AMIA Annual Symposium Proceedings, pp. 686-690, 2007.

[15] M. Pirooznia, J. Y. Yang, M. Q. Qu, and Y. Deng, "A comparative study of different machine learning methods on microarray gene expression data," BMC Genomics, vol. 9, supplement 1, article S13, 2008.

[16] M. Zucknick, S. Richardson, and E. A. Stronach, "Comparing the characteristics of gene expression profiles derived by univariate and multivariate classification methods," Statistical Applications in Genetics and Molecular Biology, vol. 7, no. 1, article 7, 2008.

[17] A. Statnikov, L. Wang, and C. F. Aliferis, "A comprehensive comparison of random forests and support vector machines for microarray-based cancer classification," BMC Bioinformatics, vol. 9, article 319, 2008.

[18] J. Önskog, E. Freyhult, M. Landfors, P. Rydén, and T. R. Hvidsten, "Classification of microarrays; synergistic effects between normalization, gene selection and machine learning," BMC Bioinformatics, vol. 12, article 390, 2011.

[19] S. Y. Kim, "Effects of sample size on robustness and prediction accuracy of a prognostic gene signature," BMC Bioinformatics, vol. 10, article 147, 2009.

[20] Y. Hoshida, "Nearest template prediction: a single-samplebased flexible class prediction with confidence assessment," PLoS One, vol. 5, no. 11, Article ID e15543, 2010.

[21] M. Chen, L. Shi, R. Kelly, R. Perkins, H. Fang, and W. Tong, "Selecting a single model or combining multiple models for microarray-based classifier development?-a comparative analysis based on large and diverse datasets generated from the MAQC-II project," BMC Bioinformatics, vol. 12, supplement 10, Article ID S3, 2011.

[22] M. Zervakis, M. E. Blazadonakis, G. Tsiliki, V. Danilatou, M. Tsiknakis, and D. Kafetzopoulos, "Outcome prediction based on microarray analysis: a critical perspective on methods," BMC Bioinformatics, vol. 10, article 53, 2009.

[23] S. Calza, P. Hall, G. Auer et al., "Intrinsic molecular signature of breast cancer in a population-based cohort of 412 patients," Breast Cancer Research, vol. 8, no. 4, article R34, 2006.

[24] C. Sotiriou, S. Y. Neo, L. M. McShane et al., "Breast cancer classification and prognosis based on gene expression profiles from a population-based study," Proceedings of the National Academy of Sciences of the United States of America, vol. 100, no. 18, pp. 10393-10398, 2003.

[25] C. Sotiriou, P. Wirapati, S. Loi et al., "Gene expression profiling in breast cancer: understanding the molecular basis of histologic grade to improve prognosis," Journal of the National Cancer Institute, vol. 98, no. 4, pp. 262-272, 2006.
[26] M. J. van de Vijver, Y. D. He, L. J. van't Veer et al., “A gene-expression signature as a predictor of survival in breast cancer," New England Journal of Medicine, vol. 347, no. 25, pp. 1999-2009, 2002.

[27] E. Huang, S. H. Cheng, H. Dressman et al., "Gene expression predictors of breast cancer outcomes," The Lancet, vol. 361, no. 9369, pp. 1590-1596, 2003.

[28] Y. Wang, J. G. M. Klijn, Y. Zhang et al., "Gene-expression profiles to predict distant metastasis of lymph-node-negative primary breast cancer," The Lancet, vol. 365, no. 9460, pp. 671679, 2005.

[29] M. Buyse, S. Loi, L. van't Veer et al., "Validation and clinical utility of a 70-gene prognostic signature for women with node-negative breast cancer," Journal of the National Cancer Institute, vol. 98, no. 17, pp. 1183-1192, 2006.

[30] M. Schmidt, D. Böhm, C. von Törne et al., "The humoral immune system has a key prognostic impact in node-negative breast cancer," Cancer Research, vol. 68, no. 13, pp. 5405-5413, 2008.

[31] L. J. van't Veer, H. Dai, M. J. van de Vijver et al., "Gene expression profiling predicts clinical outcome of breast cancer," Nature, vol. 415, no. 6871, pp. 530-536, 2002.

[32] C. Desmedt, F. Piette, S. Loi et al., "Strong time dependence of the 76-gene prognostic signature for node-negative breast cancer patients in the TRANSBIG multicenter independent validation series," Clinical Cancer Research, vol. 13, no. 11, pp. 3207-3214, 2007.

[33] L. Nanni, S. Brahnam, and A. Lumini, "Combining multiple approaches for gene microarray classification," Bioinformatics, vol. 28, no. 8, pp. 1151-1157, 2012.

[34] M. Thomassen, Q. Tan, and T. A. Kruse, "Gene expression meta-analysis identifies metastatic pathways and transcription factors in breast cancer," BMC Cancer, vol. 8, article 394, 2008.

[35] M. Thomassen, Q. Tan, and T. A. Kruse, "Gene expression meta-analysis identifies chromosomal regions and candidate genes involved in breast cancer metastasis," Breast Cancer Research and Treatment, vol. 113, no. 2, pp. 239-249, 2009.

[36] L. Breiman, "Random forests," Machine Learning, vol. 45, no. 1, pp. 5-32, 2001.

[37] T. Hastie, R. Tibshirani, and J. Friedman, The Elements of Statistical Learning: Data Mining Inference and Prediction, Springer, New York, NY, USA, 2nd edition, 2009.

[38] V. Vapnik, The Nature of Statistical Learning Theory, Springer, New York, Ny, USA, 1995.

[39] A. Dupuy and R. M. Simon, "Critical review of published microarray studies for cancer outcome and guidelines on statistical analysis and reporting," Journal of the National Cancer Institute, vol. 99, no. 2, pp. 147-157, 2007.

[40] R. Hewett and P. Kijsanayothin, "Tumor classification ranking from microarray data," BMC Genomics, vol. 9, supplement 2, article S21, 2008.

[41] G. Natsoulis, L. El Ghaoui, G. R. G. Lanckriet et al., "Classification of a large microarray data set: algorithm comparison and analysis of drug signatures," Genome Research, vol. 15, no. 5, pp. 724-736, 2005.

[42] Y. Peng, "A novel ensemble machine learning for robust microarray data classification," Computers in Biology and Medicine, vol. 36, no. 6, pp. 553-573, 2006.

[43] C. J. Xu, H. C. J. Hoefsloot, and A. K. Smilde, "To aggregate or not to aggregate high-dimensional classifiers," BMC Bioinformatics, vol. 12, article 153, 2011.

[44] S. L. Taylor and K. Kim, "A jackknife and voting classifier approach to feature selection and classification," Cancer Informatics, vol. 10, pp. 133-147, 2011. 
[45] A. Statnikov, C. F. Aliferis, I. Tsamardinos, D. Hardin, and S. Levy, "A comprehensive evaluation of multicategory classification methods for microarray gene expression cancer diagnosis," Bioinformatics, vol. 21, no. 5, pp. 631-643, 2005.

[46] A. C. Tan and D. Gilbert, "Ensemble machine learning on gene expression data for cancer classification," Appl Bioinformatics, vol. 2, supplement 3, pp. S75-S83, 2003.

[47] M. S. Sewak, N. P. Reddy, and Z. H. Duan, "Gene expression based leukemia sub-classification using committee neural networks," Bioinformatics and Biology Insights, vol. 3, pp. 8998, 2009.

[48] L. Breiman, "Bagging predictors," Machine Learning, vol. 24, no. 2, pp. 123-140, 1996.

[49] X. Wang and O. Gotoh, "Accurate molecular classification of cancer using simple rules," BMC Medical Genomics, vol. 2, article 64, 2009.

[50] H. T. Huynh, J. J. Kim, and Y. Won, "Performance comparison of SLFN training algorithms for DNA microarray classification," Advances in Experimental Medicine and Biology, vol. 696, pp. 135-143, 2011. 

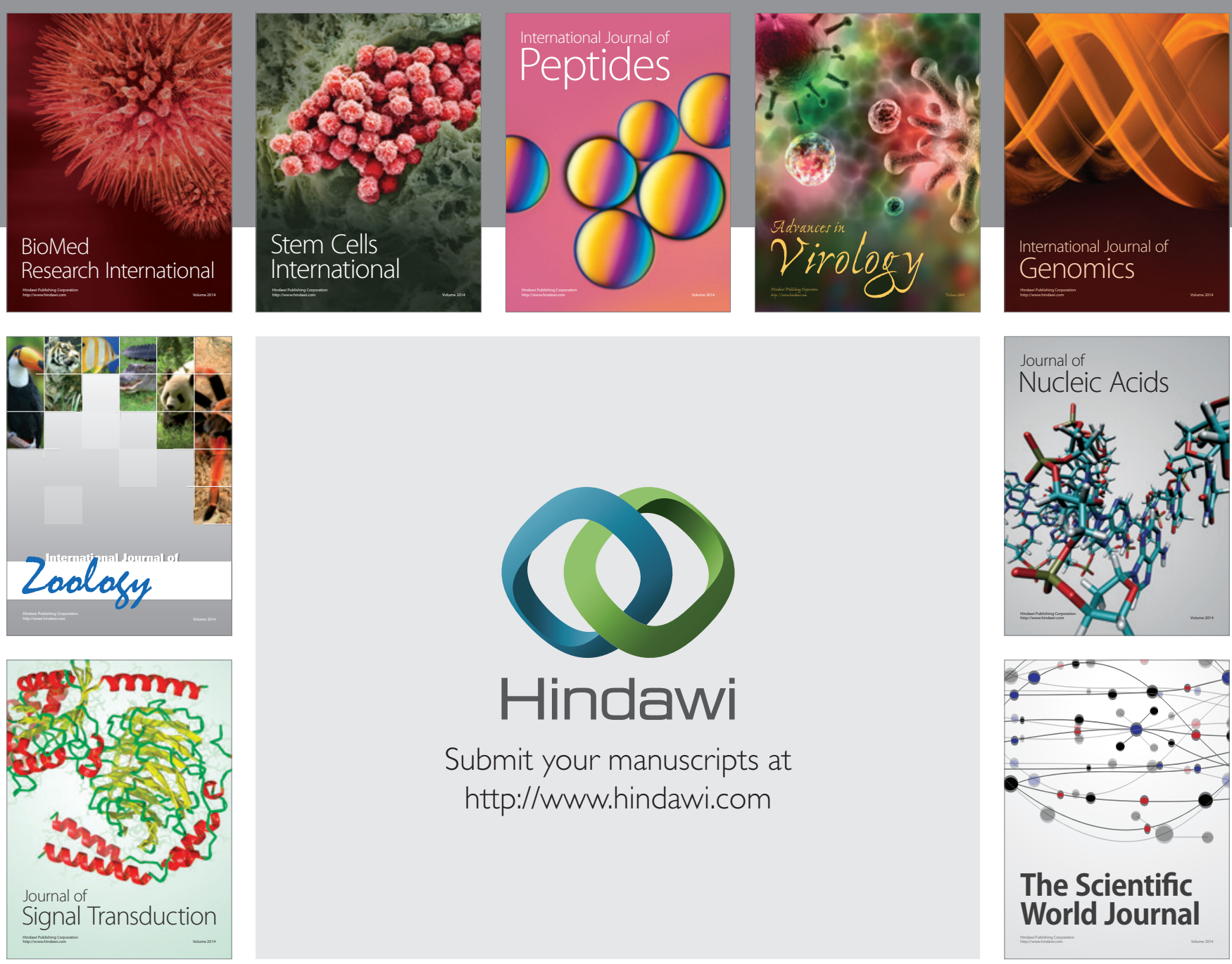

Submit your manuscripts at

http://www.hindawi.com
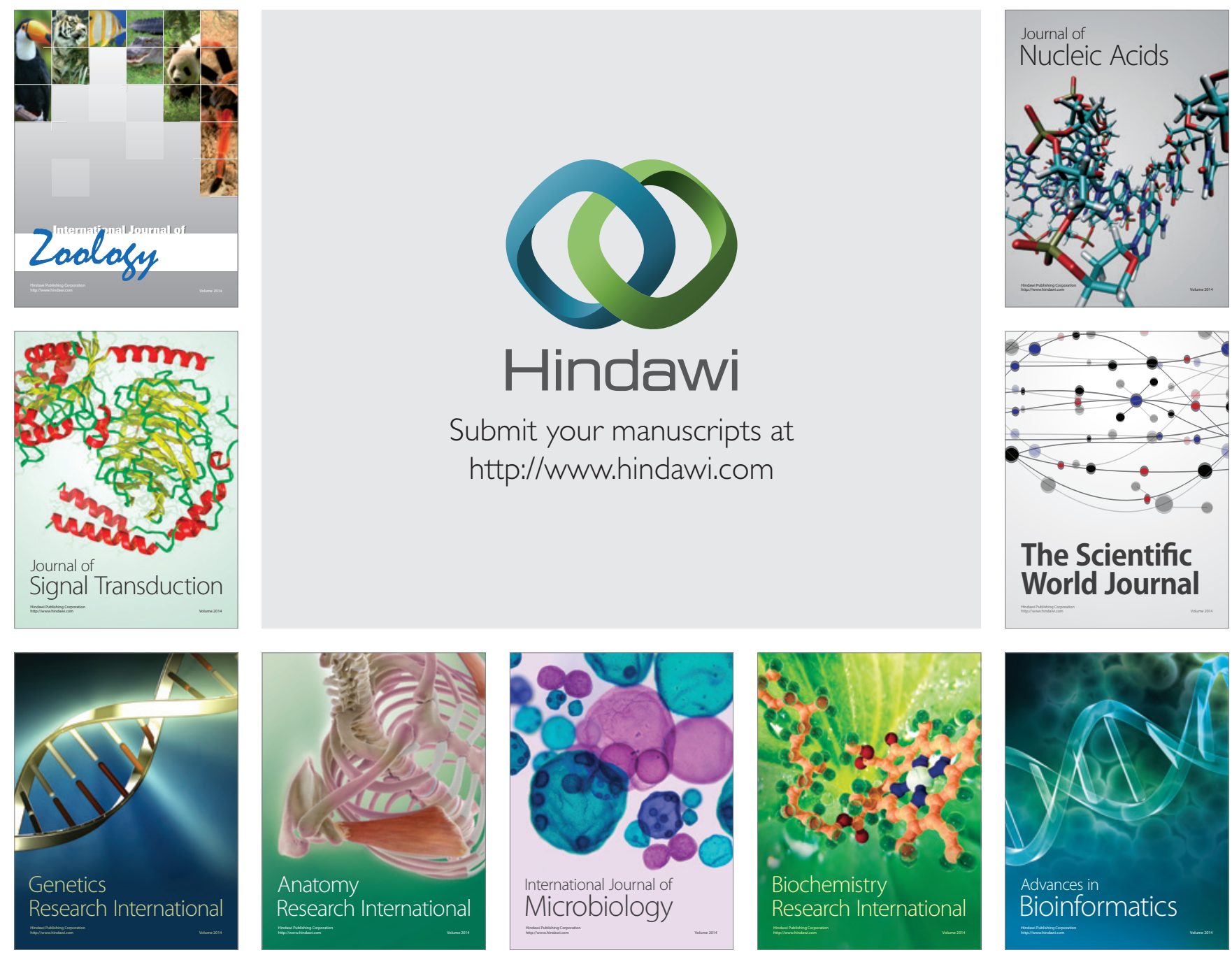

The Scientific World Journal
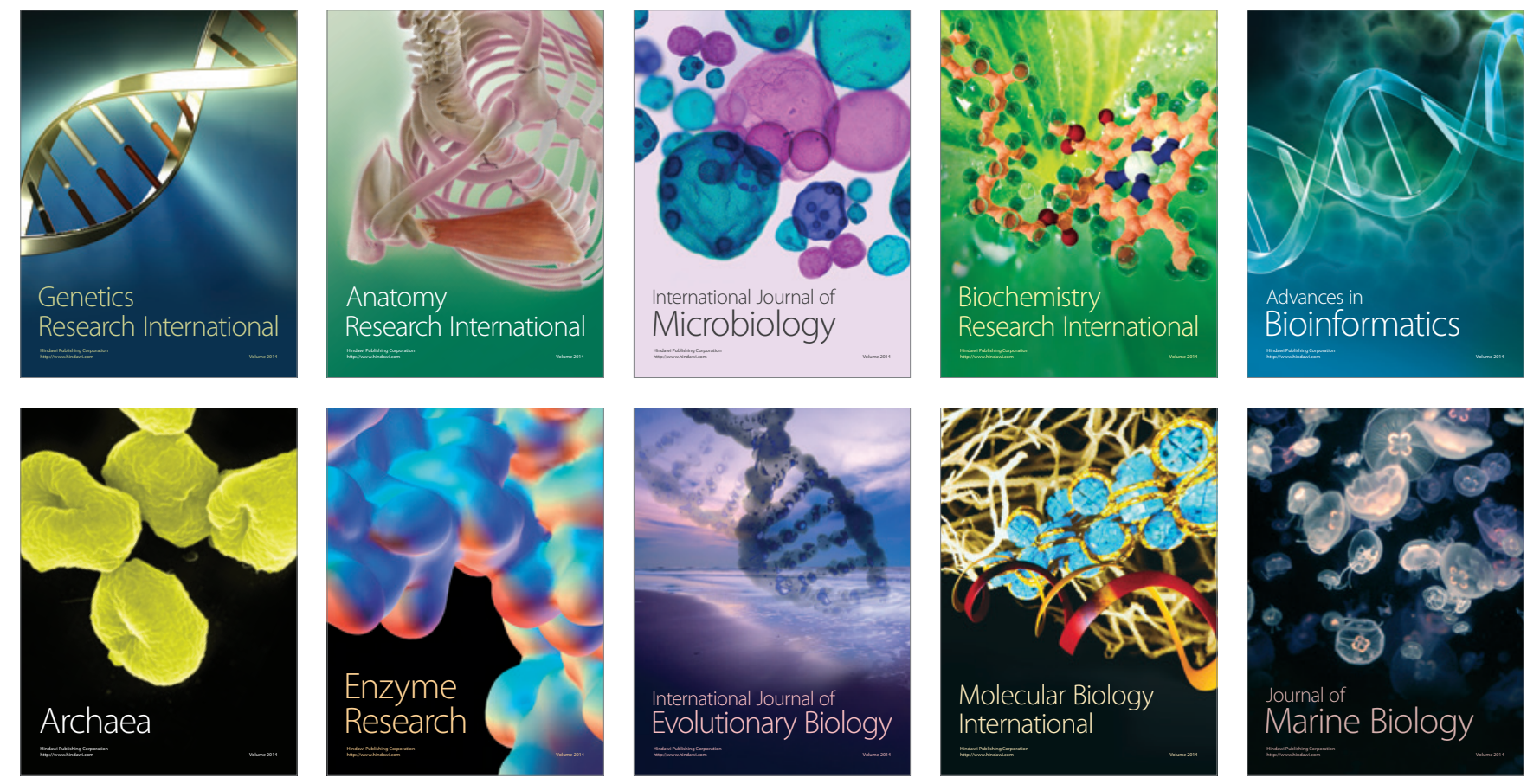